\title{
Fusion of multispectral imagery and LiDAR data for roofing materials and roofing surface conditions assessment
}

\begin{abstract}
Assessment of rooftop rainwater harvesting (RRWH) quality and suitability requires detail and reliable information on roofs. Characterization of roof surface conditions affects the quality of harvested rainwater. Nevertheless, the implementation of the system requires improvement in terms of the roof detection techniques to ensure the roof of the building is selected appropriately. Thus, the classification techniques need to be optimized to detect roof materials and roof surface conditions (new or old) with high accuracy. This study aimed to produce high precision detailed roof materials and roof surface conditions map with using high-resolution remote sensing imagery, WorldView-3 (WV3) and light detection and ranging (LiDAR) data. Three different fusion methods; layer stacking (LS), Gram-Schmidt (GS) and principal components spectral sharpening (PCSS) were explored and their performances were compared to improve the spatial and spectral richness of the image. Subsequently, the roof materials and roof surface conditions classes which include old concrete, new concrete, old metal, new metal, old asbestos and new asbestos had been discriminated by employing support vector machine (SVM) and the rule-based technique known as a decision tree (DT). Generally, generated rule-sets present a higher overall accuracy with $87 \%$, $72 \%$ and $66 \%$ for LS, GS and PCSS, respectively. For SVM classifier, the maximum accuracy recorded for LS, PCSS and GS were 70\%, 63\% and 43\% respectively. Therefore, rule-based classification via LS fusion technique was utilized to identify suitable rooftops for the development of harvested rainwater system in the urban area. Findings indicate that the degradation status of a roof in heterogenous urban environments could be determined from satellite observation and the quality of roof-based harvested rainwater affected by roofing materials and roofing surface conditions can be analysed effectively.
\end{abstract}

Keyword: Roof materials; Roof surface conditions; Fusion method; Roof-based harvested rainwater system 